\title{
Renormalization of null Wilson lines in EQCD
}

\author{
Michela D'Onofrio, ${ }^{a}$ Aleksi Kurkela ${ }^{b, c}$ and Guy D. Moore ${ }^{b}$ \\ ${ }^{a}$ Department of Physics and Helsinki Institute of Physics, University of Helsinki, \\ P.O. Box 64, FI-00014 Helsinki, Finland \\ ${ }^{b} \mathrm{Mc}$ Gill University, \\ 3600 rue University, Montréal QC H3A 2T8, Canada \\ ${ }^{c}$ CERN, Physics Department, \\ 1211 Genève 23, Switzerland \\ E-mail: michela.donofrio@helsinki.fi, a.k@cern.ch, \\ guymoore@physics.mcgill.ca
}

ABSTRACT: Radiation and energy loss of a light, high-energy parton in a perturbative Quark-Gluon Plasma is controlled by transverse momentum exchange. The troublesome infrared contributions to transverse momentum exchange can be computed on the lattice using dimensional reduction to EQCD. However a novel extended operator, the Null Wilson Line of EQCD, is involved. We compute the renormalization properties of this object's lattice implementation to next-to-leading order, which should facilitate its efficient calculation on the lattice.

KeYwords: Lattice QCD, Quark-Gluon Plasma, Wilson, 't Hooft and Polyakov loops ARXIV EPRINT: 1401.7951 


\section{Contents}

1 Introduction 1

2 Statement of the problem 3

2.1 Lattice and continuum action, Wilson line 3

2.2 Sensitivity of Wilson loop to renormalization 5

$\begin{array}{lll}3 & \text { Calculation details } & 6\end{array}$

3.1 Strategy 6

$\begin{array}{lll}3.2 & \text { Self-energies } & 7\end{array}$

3.3 Vertex corrections 8

3.4 Y-diagrams

$\begin{array}{ll}3.5 & \text { Summing it up }\end{array}$

$\begin{array}{lll}4 & \text { Discussion } & 10\end{array}$

A Scalar mass and self-coupling renormalization $\quad 11$

\section{Introduction}

The quark gluon plasma created in the laboratory [1-3] appears to be strongly coupled, in that its description requires quick thermalization and a small viscosity [4-6]. This is presumably because the temperature, and therefore the energy scale for most of the physics, is not far above the QCD transition temperature, where the coupling is large. But some of the most important probes of the medium involve the interaction of very high energy particles with the medium - hard probes, the most prominent of which is jet modification [7-9]. Even if the medium is strongly coupled, the high energy of the jet introduces a large energy scale at which QCD is weakly coupled. Therefore there is hope that jet modification can be understood perturbatively. More likely, it can be understood by treating the jet constituents and their evolution (particularly, particle splitting) perturbatively, but treating the interaction of jet constituents with the medium nonperturbatively.

The propagation of a sufficiently high energy excitation through the medium can be described in terms of a null Wilson line, and the transverse momentum exchange with the medium is related to the falloff with distance of a parallel pair of such lines [10-13]. Specifically, the probability per length to exchange transverse momentum $\Delta p_{\perp}$ is given by

$$
\frac{(2 \pi)^{2} d \Gamma}{d^{2} \Delta p_{\perp} d t} \equiv C\left(p_{\perp}\right), \quad C\left(p_{\perp}\right)=\int d^{2} x_{\perp} e^{i \mathbf{p}_{\perp} \cdot \mathbf{x}_{\perp}} C\left(x_{\perp}\right)
$$


where $C\left(x_{\perp}\right)$ is determined by a Wilson loop with two null segments of length $l$ and two transverse spatial components of length $x_{\perp}$ :

$$
\begin{aligned}
C\left(x_{\perp}\right) & =\lim _{l \rightarrow \infty}-\frac{1}{\ell} \ln \operatorname{Tr} W_{l \times x_{\perp}}, \\
W_{l \times x_{\perp}} & =\left\langle U_{(0,0,0) ;(l, 0, l)} U_{(l, 0, l) ;\left(l, \mathbf{x}_{\perp}, l\right)} U_{\left(l, \mathbf{x}_{\perp}, l\right) ;\left(0, \mathbf{x}_{\perp}, 0\right)} U_{\left(0, \mathbf{x}_{\perp}, 0\right) ;(0,0,0)}\right\rangle,
\end{aligned}
$$

where $U_{x^{\mu} ; y^{\mu}}$ are straight Wilson lines from $x^{\mu}$ to $y^{\mu}$, and the three entries are the time, transverse coordinate, and longitudinal coordinate. The Wilson loop is to be evaluated in the density matrix describing the collision, which is presumably a thermal density matrix. Knowledge of $C\left(p_{\perp}\right)$, or equivalently $C\left(x_{\perp}\right)$, is a key input into models of medium induced jet energy loss and jet modification [12-14].

The leading order perturbative form of $C\left(p_{\perp}\right)$ is fully known [15], and for momentum transfer of order of the temperature or higher $p_{\perp} \gtrsim T$, the corrections to the leading order result are suppressed by $\mathcal{O}\left(g^{2}\right)$. For a soft momentum transfer $p_{\perp} \sim g T$, however, the introduction of a soft scale forces one to use resummed perturbation theory, and the Nextto-Leading order correction arises already as an $\mathcal{O}(g)$ correction, making the physics of soft momentum transfers significantly more complicated.

However, in a remarkable paper [16], Caron-Huot has shown that for soft momentum transfers, to NLO, the Wilson loop $W_{l \times x_{\perp}}$ above can be replaced by a Wilson loop in the much simpler theory of EQCD, that is, Quantum Chromodynamics dimensionally reduced to three Euclidean dimensions, with the $A^{0}$ field converted into an adjoint scalar field which we will call $\Phi$ (roughly speaking $g_{3 \mathrm{~d}} \Phi=i A^{0}$ and $g_{3 \mathrm{~d}}^{2} \sim g^{2} T$ ). Specifically,

$$
W_{\ell \times x_{\perp}} \rightarrow \tilde{U}_{(0,0) ;(0, l)} U_{(0, l) ;\left(\mathbf{x}_{\perp}, l\right)} \tilde{U}_{\left(\mathbf{x}_{\perp}, l\right) ;\left(\mathbf{x}_{\perp}, 0\right)} U_{\left(\mathbf{x}_{\perp}, 0\right) ;(0,0)} .
$$

There is now no time coordinate, only the transverse and $z$ coordinates. The complication is that the Wilson lines which replace the null lines in the 4-D version of $W$ are modified, still containing the descendant of the $A^{0}$ field, which enters in the definition of $\tilde{U}$ :

$$
\tilde{U}_{(0,0) ;(0, l)}=\operatorname{Pexp} \int_{0}^{l} d z T_{a}\left(i A_{z}^{a}+g_{3 \mathrm{~d}} \Phi^{a}\right) .
$$

The representation matrices $T_{a}$ should be in the same representation as the propagating particle, which we will label $R$ (typically the fundamental or adjoint representation). The relative factor of $g_{3 \mathrm{~d}}$ is because we absorbed a $g_{3 \mathrm{~d}}$ factor in defining $\Phi$. The relative phase - $A_{z}$ enters with an $i$ and $\Phi$ does not - is because $\Phi$ is a Euclidean continuation of $A^{0}$ and the $i$ factor is absorbed in the Wick rotation. The overall sign is reversed in $\tilde{U}_{\left(\mathbf{x}_{\perp}, l\right) ;\left(\mathbf{x}_{\perp}, 0\right)}$. We will call this modified Wilson line the null Wilson line of EQCD.

Perturbation theory fails near the QCD crossover because the theory is genuinely strongly coupled there. But it is possible that the failure of perturbation theory at a few times the crossover temperature arises because the 3D theory is strongly coupled, while the short-distance physics involved in dimensional reduction is not [17]. In this case, a nonperturbative treatment of the 3D theory may still give useful information about QCD at the highest temperatures achieved in heavy ion collisions. If true, then the nonperturbative nature in the interaction of a jet parton with the medium is captured by the EQCD value 
of $C\left(p_{\perp}\right)$, which can be measured on the lattice. With this motivation, there has been an upswing in interest, recently, in studying the Wilson loop and $C\left(x_{\perp}\right)$ in EQCD on the lattice [18]. The relation between continuum thermal QCD and continuum EQCD is known to high perturbative order [17, 19-22], and the matching of the action, and some operators, between continuum and lattice EQCD is known to order $g_{3 \mathrm{~d}}^{2} a[23]{ }^{1,2}$ But the Wilson line in eq. (1.4) is a new operator and its lattice implementation has not been studied beyond the tree level. In practice it is challenging to make lattice studies quantitatively reliable without a calculation of the $\mathcal{O}\left(g_{3 \mathrm{~d}}^{2} a\right)$ renormalization of the null Wilson line operator. This is true even if the lattice spacing is taken very small, if one is simultaneously interested in $C\left(x_{\perp}\right)$ at short distances; as we will argue below, the corrections arising from the Wilson operator scale as the larger of $g_{3 \mathrm{~d}}^{2} a$ and $a / x_{\perp}$. Indeed, the first efforts to numerically determine the $C\left(p_{\perp}\right)$ by Panero, Rummukainen, and Schäfer [18] show how it is challenging to make contact with perturbation theory at $p_{\perp} \gg g T$, corresponding to small spatial separations. Therefore we believe that a study of $\mathcal{O}(a)$ corrections to the null Wilson line operator are essential to the success of this program. We carry out this calculation in the remainder of the paper.

In the next section we set up the problem, by writing the Lagrangian of EQCD and an expression for the Wilson line in the continuum and the lattice, highlighting what is needed in an NLO matching calculation. The section also shows why the $\mathcal{O}(a)$ correction becomes more important at small $x_{\perp}$. The body of the calculation appears in section 3 , which explains how to handle lattice diagrams with null Wilson lines, and tabulates the (Feynman gauge) contribution of each relevant diagram. We close with a brief discussion.

\section{Statement of the problem}

\subsection{Lattice and continuum action, Wilson line}

EQCD is the theory of a 3 -dimensional $\mathrm{SU}(N)$ gauge field $A^{i}$ with field strength $F^{i j} \equiv$ $F_{a}^{i j} T^{a}$, together with an adjoint scalar $\Phi \equiv \Phi^{a} T_{a}$ [with $T_{a}$ the fundamental representation group generators normalized such that $\left.\operatorname{Tr} T_{a} T_{b}=\delta_{a b} / 2\right]$. Writing the path integral as $\int \mathcal{D}[A, \Phi] \exp \left(-S_{\mathrm{EQCD}}\right)$, the most general super-renormalizable action ${ }^{3}$ in the continuum is

$$
S_{\mathrm{EQCD}, \mathrm{c}}=\int d^{3} x\left(\frac{1}{2 g_{3 \mathrm{~d}}^{2}} \operatorname{Tr} F^{i j} F^{i j}+\operatorname{Tr} D^{i} \Phi D^{i} \Phi+m_{\mathrm{D}}^{2} \operatorname{Tr} \Phi^{2}+\lambda_{1}\left(\operatorname{Tr} \Phi^{2}\right)^{2}+\lambda_{2} \operatorname{Tr} \Phi^{4}\right),
$$

where we have not shown the counterterm which subtracts UV divergences from the $\operatorname{Tr} \Phi^{2}$ term. ${ }^{4}$ The three dimensional theory corresponds to the dimensionally reduced four di-

\footnotetext{
${ }^{1}$ In the 3-D theory the gauge coupling $g^{2}$ is dimensionful, carrying units of energy or inverse length; so $g^{2} a$ is a dimensionless quantity.

${ }^{2}$ Actually the $\mathcal{O}\left(g^{2} a\right)$ matching between continuum and lattice EQCD is incomplete; the mass parameter of the $\Phi$ field is only known to two-loop order [24], which is $\mathcal{O}\left(a^{0}\right)$. Improving this parameter to $\mathcal{O}\left(g^{2} a\right)$ will require a 3 -loop calculation, though there is a way to determine the matching within numerical EQCD simulations, which we outline in appendix A.

${ }^{3}$ The Lagrangian could in addition contain a $\operatorname{Tr} \Phi^{3}$ term, but at zero baryon number chemical potential it is forbidden by the charge conjugation symmetry.

${ }^{4}$ For the exact form of the counterterm see, e.g., eq. (2.8) of [26].
} 
mensional QCD along a matching curve, specifying the values of the parameters of EQCD as a function of four-dimensional parameters: $g, T$, and $N$ and the number and masses of quark species $N_{f}$ and $m_{i}$. For explicit expressions see for example eq. (5.2)-(5.5) of [27]. For quark mass dependence, see [28].

It is customary to introduce dimensionless versions of the mass and scalar coupling terms, by defining ${ }^{5}$

$$
y \equiv \frac{m_{\mathrm{D}}^{2}\left[\mu_{\overline{\mathrm{MS}}}=g_{3 \mathrm{~d}}^{2}\right]}{g_{3 \mathrm{~d}}^{4}}, \quad x_{1}=\frac{\lambda_{1}}{g_{3 \mathrm{~d}}^{2}}, \quad x_{2}=\frac{\lambda_{2}}{g_{3 \mathrm{~d}}^{2}} .
$$

The corresponding lattice theory, with lattice spacing $a$, is defined in terms of the link matrices $U_{i}(x)=U_{x ; x+a \hat{i}}$ and the lattice scalar field $\Phi_{\mathrm{L}}$. The lattice action is

$$
\begin{aligned}
S_{\mathrm{EQCD}, \mathrm{L}}= & \frac{2 N}{Z_{g} g_{3 \mathrm{~d}}^{2} a} \sum_{x, i>j}\left(1-\frac{1}{N} \operatorname{Tr} \square_{x, i j}\right) \\
& +2 Z_{\Phi} \sum_{x, i} \operatorname{Tr}\left(\Phi_{\mathrm{L}}^{2}(x)-\Phi_{\mathrm{L}}(x) U_{i}(x) \Phi_{\mathrm{L}}(x+a \hat{i}) U_{i}^{\dagger}(x)\right) \\
& +\sum_{x} Z_{4}\left[\left(x_{1}+\delta x_{1}\right) \operatorname{Tr} \Phi_{\mathrm{L}}^{4}+\left(x_{2}+\delta x_{2}\right)\left(\operatorname{Tr} \Phi_{\mathrm{L}}^{2}\right)^{2}\right]+Z_{2}(y+\delta y) \operatorname{Tr} \Phi_{\mathrm{L}}^{2}, \\
\square_{x, i j} \equiv & U_{i}(x) U_{j}(x+a \hat{i}) U_{i}^{\dagger}(x+a \hat{j}) U_{j}^{\dagger}(x)
\end{aligned}
$$

and the lattice implementation of $\tilde{U}$ is ${ }^{6}$

$$
\tilde{U}_{(0,0) ;(0, n a)}=\prod_{m=0}^{n-1} \exp \left(Z T_{R}^{a} \Phi_{\mathrm{L}}^{a}(m a \hat{z})\right) U_{z, R}(m a \hat{z}),
$$

for a Wilson line in the $R$ representation. Note that there is no factor of $i$ in $\exp \left(Z \Phi_{\mathrm{L}}\right)$, which is not a unitary matrix.

The value of the scalar field wave function normalization $Z_{\Phi}$ is actually a free choice in implementing the lattice theory, corresponding to the normalization choice for the lattice scalar field. For instance, Panero, Rummukainen, and Schäfer [18] choose $Z_{\Phi}=g_{3 \mathrm{~d}}^{2} a Z_{g}$ (which they call $6 / \beta$ ). Another sensible choice would be $Z_{\Phi}=1 /\left(g_{3 \mathrm{~d}}^{2} a Z_{g}\right.$ ), so the lattice spacing enters the action as a common multiplicative factor. We will not choose a specific prescription in this paper. Instead, we focus on the combinations $Z_{g}, Z^{2} / Z_{\Phi}, Z_{2} / Z_{\Phi}$, and $Z_{4} / Z_{\Phi}^{2}$, which are invariant under this normalization freedom. At tree level we would have $Z_{g}=1, Z^{2} / Z_{\Phi}=g_{3 \mathrm{~d}}^{2} a=Z_{4} / Z_{\Phi}^{2}$ and $Z_{2} / Z_{\Phi}=g_{3 \mathrm{~d}}^{4} a^{2}$. The coefficients $Z_{g}, Z_{2} / Z_{\Phi}, Z_{4} / Z_{\Phi}^{2}$, $\delta x_{1,2}$ and $\delta y$ are already known. For completeness we list their values in appendix A. Our goal is to determine the remaining unknown parameter $Z^{2} / Z_{\Phi}$, which controls the renormalization of the null Wilson line of EQCD.

\footnotetext{
${ }^{5}$ Note that, for $\mathrm{SU}(2)$ or $\mathrm{SU}(3)$, the $\operatorname{Tr} \Phi^{4}$ and $\left(\operatorname{Tr} \Phi^{2}\right)^{2}$ terms are not independent, as $\operatorname{Tr} \Phi^{4}=$ $\left(\operatorname{Tr} \Phi^{2}\right)^{2} / 2$ for these groups. So in these cases one of the scalar terms can be eliminated in favor of the other.

${ }^{6}$ Actually the implementation shown here is not unique; for instance, one can also replace $\exp \left(Z \Phi_{\mathrm{L}}\right) \rightarrow$ $Z_{0}+Z_{1} \Phi_{\mathrm{L}}$, avoiding the need to exponentiate. But there are advantages to the exponential choice; for instance, $Z_{0}, Z_{1}$ are already nontrivial in an abelian theory, for which $Z$ takes its tree-level value. We will only consider the exponential choice here.
} 


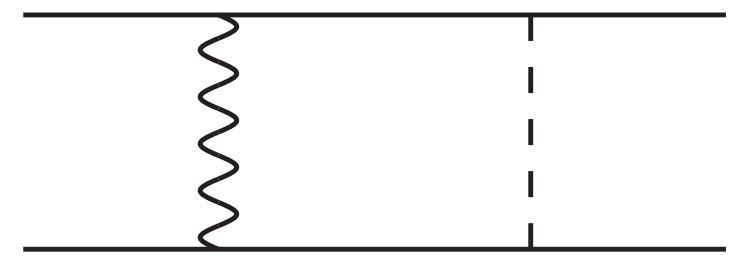

Figure 1. Diagrams giving rise to the leading-order contribution to $C\left(x_{\perp}\right)$. Wilson line (solid), showing the exchange between lines of an $A$ field (wiggly) or a $\Phi$ field (dashed)

\subsection{Sensitivity of Wilson loop to renormalization}

Since we are interested in the $l$-dependence of $\operatorname{Tr} W$ when $l$ is large, we can ignore contributions from the ends and corners of the Wilson loop and focus on correlations between the long edges. We are also only interested in the $x_{\perp}$ dependence of $C\left(x_{\perp}\right)$, since any $x_{\perp}$-independent piece does not enter in $C\left(p_{\perp}\right)$. Therefore we need only consider diagrams with at least one line connecting the null Wilson lines. At lowest order there are two, involving the exchange of an $A_{z}$ or a $\Phi$ line, as illustrated in figure 1. Because the $A_{z}$ fields attach with factors of $i,-i$ while the $\Phi$ fields attach with factors of $1,-1$, the contributions are of opposite sign. In the continuum they are

$$
C_{\mathrm{LO}}\left(x_{\perp}\right)=\int_{-\infty}^{\infty} d z\left\langle A_{z}\left(x_{\perp}, z\right) A_{z}(0)-g_{3 \mathrm{~d}}^{2} \Phi\left(x_{\perp}, z\right) \Phi(0)\right\rangle \Rightarrow \frac{C\left(p_{\perp}\right)}{C_{R}}=\frac{g_{3 \mathrm{~d}}^{2}}{p_{\perp}^{2}}-\frac{g_{3 \mathrm{~d}}^{2}}{p_{\perp}^{2}+m_{\mathrm{D}}^{2}},
$$

while on the lattice we find (defining, as usual $\left.U_{i}(x)=\exp \left(1+i a A_{i}(x+a \hat{i} / 2)\right)\right)$

$C_{\mathrm{LO}}\left(x_{\perp}\right)=\frac{1}{a} \sum_{n}\left\langle a^{2} A_{z}\left(x_{\perp}, n a\right) A_{z}(0)-Z^{2} \Phi\left(x_{\perp}, n a\right) \Phi(0)\right\rangle \Rightarrow \frac{C\left(p_{\perp}\right)}{C_{R}}=\frac{Z_{g} g^{2}}{\tilde{p}_{\perp}^{2}}-\frac{Z^{2} / a Z_{\Phi}}{\tilde{p}_{\perp}^{2}+m_{\mathrm{D}}^{2}}$.

Here $\tilde{p}_{x}^{2} \equiv \sin ^{2}\left(p_{x} a / 2\right) /(a / 2)^{2}$ is the lattice propagator, and $C_{R}$ is the quadratic Casimir in the representation $R$ of the Wilson loop.

The important feature of eq. (2.6) is that the two terms approximately cancel at large $p_{\perp}$, up to subleading $m_{\mathrm{D}}^{2} / p_{\perp}^{4}$ corrections. The presence of the lattice propagator in eq. (2.7) does not change this cancellation. Of course this cancellation does not persist at higher loop order; but because the theory is super-renormalizable, each loop order gives weaker large- $p_{\perp}$ behavior. Indeed, at NLO the large $p_{\perp}$ behavior is $\mathcal{O}\left(g_{3 \mathrm{~d}}^{4} / p_{\perp}^{3}\right)[16]$.

The problem is that the renormalization of $Z$ which is not taken into account in a lattice calculation will spoil the cancellation in eq. (2.7), giving rise to uncanceled $1 / p_{\perp}^{2}$ large- $p_{\perp}$ behavior - specifically, a contribution of $\left(1-Z_{\text {used }}^{2} / Z_{\text {correct }}^{2}\right) g_{3 \mathrm{~d}}^{2} / p_{\perp}^{2}-$ in eq. (2.7). Therefore the short-distance or large- $p_{\perp}$ behavior is especially sensitive to errors in the Wilson line renormalization constant $Z$. Assuming $Z_{\text {used }}^{2} / Z_{\text {correct }}^{2}=1+\mathcal{O}\left(g_{3 \mathrm{~d}}^{2} a\right)$, the relative error is of order $\left(g_{3 \mathrm{~d}}^{4} a / p_{\perp}^{2}\right) /\left(g_{3 \mathrm{~d}}^{4} / p_{\perp}^{3}\right) \sim a p_{\perp}$, corresponding to a $a / x_{\perp}$ relative error in $C\left(x_{\perp}\right)$. Therefore the need to renormalize the Wilson line operator increases at small separation, scaling as the inverse separation of the Wilson lines in lattice units. For instance, if the Wilson lines are separated by $N_{\perp}$ lattice spacings in the transverse direction, the $\mathcal{O}(a)$ corrections are $\mathcal{O}\left(1 / N_{\perp}\right)$, no matter how small the lattice spacing may be. Finding the $\mathcal{O}(a)$ 

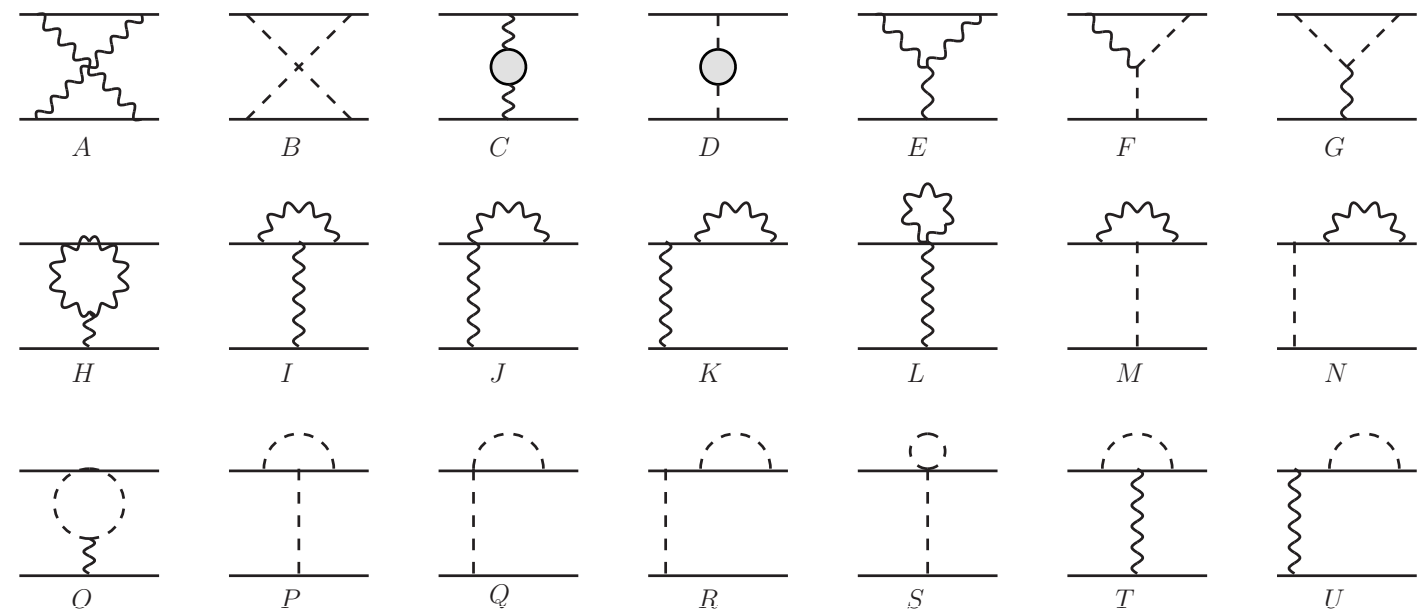

Figure 2. Diagrams needed at next-to-leading order. Solid lines are the Wilson lines, wiggly lines are $A$ fields, dashed lines are $\Phi$ fields, blobs are self-energies. Each diagram implicitly also represents the same diagram reflected right-left or top-bottom.

correction to $Z$ will improve this behavior to $1 / N_{\perp}^{2}$, an important correction for realistic values $N_{\perp} \sim 5$.

\section{Calculation details}

\subsection{Strategy}

The matching calculation consists of computing $C\left(p_{\perp}\right) / C_{R}$ at NLO within continuum and lattice EQCD, and fixing the coefficients of the lattice theory such that the calculations agree to all orders in $g_{3 \mathrm{~d}}$ and $\lambda_{i}$ and up to the desired order in $a$, here $\mathcal{O}(a)$. As usual, once the coefficients are fixed at one order, the infrared behavior is automatically the same at the next order, since the infrared behaviors of the theories coincide by construction. Then it is the difference in the ultraviolet region of any loops which must be calculated. As usual, such behavior can be understood in terms of a renormalization of the parameters of the theory appearing in diagrams of lower order.

Again we only need diagrams with at least one line running between the null Wilson lines. There are a number of NLO diagrams, see figure 2. Fortunately, both propagators in diagrams $A$ and $B$ must be infrared (since they connect spatially well-separated Wilson lines), so they do not contribute to UV renormalization. In Feynman gauge (which we will use throughout), diagrams $E, H$, and $O$ are zero. Diagrams $J, L, Q, S$ have no continuum analog; they arise because $U_{z}=\exp \left(i a A_{z}\right)$ and $\exp (Z \Phi)$ are nonlinear in $A_{z}$ and $\Phi$. But the form of the lattice Wilson line, eq. (2.5), does not contain anything which would introduce mixed $A_{z}, \Phi$ vertices on the Wilson line, so there are no mixed-field analogs of diagrams $H, J, L, O, Q, S$.

Our strategy will be the following. Since only the UV behavior of diagrams is relevant, we can ignore $m_{\mathrm{D}}$ and treat the propagators to be

$$
\left\langle A_{z} A_{z}(p)\right\rangle=\frac{Z_{g} g_{3 \mathrm{~d}}^{2}}{\tilde{p}^{2}}, \quad\left\langle\Phi_{\mathrm{L}} \Phi_{\mathrm{L}}(p)\right\rangle=\frac{a Z_{\Phi}^{-1}}{\tilde{p}^{2}} .
$$


In this case, for a soft momentum $p_{\perp} \ll 1 / a$ running between the Wilson lines, we extract all $1 / p_{\perp}^{2}$ contributions, and choose the value of $Z^{2} / Z_{\Phi}$ such that they cancel, as they do in the continuum according to eq. (2.6).

\subsection{Self-energies}

Diagrams $C$ and $D$ have been calculated [23]. Indeed, diagram $C$ makes up the principal contribution to $Z_{g}$ the gauge action renormalization. For this reason, we reproduce here the expression, from [23], for $Z_{g}$. Ref. [23] finds:

$$
Z_{g}^{-1}=1+2\left(Z_{\Phi}-1\right)+2\left(V_{A, L}-V_{A, c}\right)+\frac{\left(\pi_{A, L}-\pi_{A, c}\right)}{p^{2}},
$$

where $V_{A, L}$ and $V_{A, c}$ are the one loop contributions to the three-point gauge-scalar vertex on the lattice and in continuum, respectively, while $\pi_{A, L}$ and $\pi_{A, c}$ are the corresponding gauge self-energies. While $Z_{g}^{-1}$ itself is gauge invariant, the individual contribution of each term to $Z_{g}^{-1}$ is gauge dependent. Nevertheless, we only need the answer in Feynman gauge, in which they read:

$$
\begin{aligned}
2\left(Z_{\Phi}-1\right)+2\left(V_{A, L}-V_{A, c}\right) & =\frac{g_{3 \mathrm{~d}}^{2} a}{4}\left[8 C_{A} \frac{\xi}{4 \pi}\right] \\
\frac{\left(\pi_{A, L}-\pi_{A, c}\right)}{p^{2}} & =\frac{g_{3 \mathrm{~d}}^{2} a}{4}\left(C_{A}\left\{\frac{1}{6} \frac{\Sigma}{4 \pi}-\frac{2}{3} \frac{\xi}{4 \pi}\right\}+\left[\frac{4 C_{F}}{3}-\frac{C_{A}}{3}+\frac{13 C_{A}}{3} \frac{\xi}{4 \pi}\right]\right) .
\end{aligned}
$$

We have written out color factors in terms of the fundamental Casimir $C_{F}$ and dimension $d_{F}$, and the adjoint Casimir $C_{A}$ and dimension $d_{A}$ : in $\mathrm{SU}(N)$ theory these are $d_{F}=N$, $C_{F}=\left(N^{2}-1\right) /(2 N), C_{A}=N$ and $d_{A}=\left(N^{2}-1\right)$. The constants appearing here are are

$$
\frac{\Sigma}{4 \pi a} \equiv \int_{-\pi / a}^{\pi / a} \frac{d^{3} p}{(2 \pi)^{3}} \frac{1}{\tilde{p}^{2}}, \quad \frac{\xi a}{4 \pi} \equiv \int_{-\pi / a}^{\pi / a} \frac{d^{3} p}{(2 \pi)^{3}} \frac{1}{\left(\tilde{p}^{2}\right)^{2}}-\int_{-\infty}^{\infty} \frac{d^{3} p}{(2 \pi)^{3}} \frac{1}{\left(p^{2}\right)^{2}}
$$

where in the latter expression we implicitly IR regulate both integrals in the same way; numerically $\xi=0.152859324966101$ and $\Sigma=3.17591153562522$. These are the only constants we will need in the remainder of the calculation.

The terms in curly brackets in eq. (3.4) arise from gauge self-energy diagrams with $\Phi$ running in the loops, and are, in fact, independent of the gauge parameter. The terms in square brackets arise from pure gauge self-energy diagrams ${ }^{7}$ and in a general gauge, would acquire a dependence on the gauge parameter.

In Feynman gauge, the contribution from diagram $\mathrm{C}$ to $C\left(p_{\perp}\right) / C_{R}$ reads simply

$$
\text { Diagram } C=-g_{3 \mathrm{~d}}^{2} \frac{\left(\pi_{A, L}-\pi_{A, c}\right)}{\left(p_{\perp}^{2}\right)^{2}},
$$

so that if we consider the sum of the self-energy diagram and the leading-order $A_{z}$ exchange contribution, the self energy contributions cancel and leave only the parts of $Z_{g}$ which arise

\footnotetext{
${ }^{7}$ It may look strange that the pure-glue self-energy contains a rather large term proportional to $C_{F}$. This is a tadpole-type contribution, which is dependent on the choice to implement the lattice link operators as fundamental-representation matrices.
} 
from other sources:

$$
\frac{Z_{g} g_{3 \mathrm{~d}}^{2}}{p_{\perp}^{2}}+\text { Diagram } C=\frac{g_{3 \mathrm{~d}}^{2}}{p_{\perp}^{2}}\left(1-\frac{g_{3 \mathrm{~d}}^{2} a C_{A}}{4} 8 \frac{\xi}{4 \pi}\right) .
$$

The scalar self-energy is also computed in ref. [23], where it is responsible for the quantity called $Z_{\Phi}$ there. Re-computing to convert from Landau to Feynman gauge using the result for the self-energy in ref. [25], we find the sum of the tree level and self-energy $\Phi$ exchange diagrams to give

$$
-\frac{g_{3 \mathrm{~d}}^{2} Z^{2} / a Z_{\Phi}}{\tilde{p}_{\perp}^{2}}+\text { Diagram } D=-\frac{Z^{2} / a Z_{\Phi}}{\tilde{p}_{\perp}^{2}}\left(1+\frac{g_{3 \mathrm{~d}}^{2} a C_{A}}{4}\left[8 \frac{\xi}{4 \pi}+\frac{2}{3} \frac{\Sigma}{4 \pi}\right]\right) .
$$

\subsection{Vertex corrections}

Next consider diagrams $M, N$. Here it is relevant that eq. (1.2) involves $\ln \operatorname{Tr} W$, not just its trace. In an abelian theory the Wilson loop is the exponential of all 1-propagator corrections, ${ }^{8}$ which means that the abelian parts of diagrams $I-N$ and $P-U$ are absorbed when we take the log. Only the nonabelian parts of these diagrams contribute. The group theory factor in diagram $N$ is $T^{a} T^{a} T^{b} T^{b}=C_{R}^{2}$, the product of the group factors for each line. Therefore $N$ is abelian. Diagram $M$ involves $T^{a} T^{b} T^{a} T^{b}=C_{R}\left(C_{R}-C_{A} / 2\right)$; the $C_{R}^{2}$ piece is the abelian part, the $C_{R} C_{A}$ piece is the nonabelian part we need. Label the momenta on the $\Phi_{\mathrm{L}}$ and $A_{z}$ lines $p$ and $q$ respectively. The $\Phi_{\mathrm{L}}$ line can attach anywhere on each Wilson line. The sum over locations on the lower Wilson line gives a factor $\ell / a$, which cancels the $a$ in the propagator and gives the $\ell$ which should be canceled in eq. (1.2). The sum over the location of the upper Wilson line gives a factor $\delta\left(a p_{z}\right)$ which ensures that $p$ is purely transverse; the $\Phi$ line then gives rise to the $Z^{2} / a Z_{\Phi} / \tilde{p}_{\perp}^{2}$ term found in eq. (2.7).

Next we sum over the attachment positions of the $A_{z}$ propagator. It is most convenient to consider the link matrix $U_{z}(x)$ to "live" at the center of the link $x+a \hat{z} / 2$. In this case, for a line momentum $q$, the sum over attachment locations gives

$$
\left(a \sum_{n=0}^{\infty} e^{i q_{z}(n+1 / 2) a}\right)\left(a \sum_{m=0}^{\infty} e^{-i q_{z}(-m-1 / 2) a}\right),
$$

where the first (second) term sums over the attachment of the front (back) vertex, relative to where the $\Phi_{\mathrm{L}}$ attaches. The sum is easily performed by splitting off the first term and shifting the remaining terms:

$$
\begin{aligned}
& a \sum_{n=0}^{\infty} e^{i q_{z}(n+1 / 2) a}=a e^{i q_{z} a / 2}+a e^{i q_{z} a} \sum_{n=0}^{\infty} e^{i q_{z}(n+1 / 2) a} \Rightarrow \\
& a \sum_{n=0}^{\infty} e^{i q_{z}(n+1 / 2) a}=\frac{a e^{i q_{z} a / 2}}{1-e^{i q_{z} a}}=\frac{i a}{2 \sin \left(q_{z} a / 2\right)} \equiv \frac{i}{\tilde{q}_{z}} .
\end{aligned}
$$

This term will always arise when summing over the location of an $A_{z}$ attachment which must be to the right of a $\Phi_{\mathrm{L}}$ attachment on the Wilson line. Therefore it makes sense to

\footnotetext{
${ }^{8}$ Here it is essential that both the $A$ and $\Phi$ field attachments are implemented via exponentials in eq. (2.5). In the implementation suggested in footnote 6 , exponentiation would fail for the $\Phi_{\mathrm{L}}$ field.
} 
define it as the Feynman rule for the propagator of the Wilson line between a $\Phi_{\mathrm{L}}$ and an $A_{z}$ attachment. The corresponding continuum expression is $i / q_{z}$.

The group theoretical issues in treating diagrams $I, J, K, L$ are similar. Each involves a factor $C_{R}^{2}$ and a factor $-C_{R} C_{A}$, with coefficient $-1 / 2,-1 / 4,0$, and $-1 / 6$ for $I, J, K$, and $L$ repectively. The sum over the attachment points in diagram $I$ is similar to that in diagram $M$, except that the attachments must be separated an integer distance. They therefore involve the sum

$$
a \sum_{n=1}^{\infty} e^{i q_{z} n a}=e^{i q_{z} a}\left(1+a \sum_{n=1}^{\infty} e^{i q_{z} n a}\right)=\frac{i e^{i q_{z} a / 2}}{\tilde{q}_{z}}=\frac{i \cos \frac{q_{z} a}{2}}{\tilde{q}_{z}}-\frac{a}{2} \equiv \frac{i q_{z}}{\tilde{q}_{z}}-\frac{a}{2} .
$$

The sum of the nonabelian contributions from diagrams $I, J, K, L$ is therefore

$$
\begin{aligned}
I+J+K+L & =-C_{A} g_{3 \mathrm{~d}}^{2} \frac{C_{R} g_{3 \mathrm{~d}}^{2}}{\tilde{p}_{\perp}^{2}} \int_{-\pi / a}^{\pi / a} \frac{d^{3} q_{\perp}}{(2 \pi)^{3}} \frac{1}{\tilde{q}^{2}}\left(\left[\frac{q_{z}}{\tilde{q}_{z}}+\frac{i a}{2}\right]_{I}^{2}-i a\left[\frac{q_{z}}{\tilde{q}_{z}}+\frac{i a}{2}\right]_{J}-\left[\frac{a^{2}}{3}\right]_{L}\right) \\
& =\frac{C_{R} g_{3 \mathrm{~d}}^{2}}{\tilde{p}_{\perp}^{2}}\left(-C_{A} g_{3 \mathrm{~d}}^{2}\right) \int_{-\pi / a}^{\pi / a} \frac{d^{3} q_{\perp}}{(2 \pi)^{3}} \frac{1}{\tilde{q}^{2}}\left(\frac{q_{z}^{2}}{\frac{\tilde{q}_{z}^{2}}{2}}-\frac{a^{2}}{12}\right) .
\end{aligned}
$$

We can rewrite

$$
\underline{q}_{z}^{2} \equiv \cos ^{2} \frac{q_{z} a}{2}=1-\frac{a^{2} \tilde{q}_{z}^{2}}{4}
$$

and therefore

$$
I+J+L=-C_{A} g_{3 \mathrm{~d}}^{2} \frac{C_{R} g_{3 \mathrm{~d}}^{2}}{\tilde{p}_{\perp}^{2}} \int_{-\pi / a}^{\pi / a} \frac{d^{3} q_{\perp}}{(2 \pi)^{3}} \frac{1}{\tilde{q}^{2}}\left(\frac{1}{\tilde{q}_{z}^{2}}-\frac{a^{2}}{3}\right)
$$

The calculation of $P, Q, R, S$ proceeds similarly and the result is in fact identical except for a factor of $\left(-Z^{2} / a Z_{\Phi}\right)^{2}$, which is 1 at the level of precision needed in the current calculation. On the other hand, diagrams $M$ and $T$ each give

$$
M=T=+C_{A} g_{3 \mathrm{~d}}^{2} \frac{C_{R} g_{3 \mathrm{~d}}^{2}}{\tilde{p}_{\perp}^{2}} \int_{-\pi / a}^{\pi / a} \frac{d^{3} q_{\perp}}{(2 \pi)^{3}} \frac{1}{\tilde{q}^{2}} \frac{1}{\tilde{q}_{z}^{2}} .
$$

These cancel the like factors from $I, J, L, P, Q, S$, so that all vertex correction diagrams add to

$$
I-N \text { plus } P-U=\frac{C_{R} g_{3 \mathrm{~d}}^{2}}{\tilde{p}_{\perp}^{2}} C_{A} g_{3 \mathrm{~d}}^{2} \int_{-\pi / a}^{\pi / a} \frac{d^{3} q_{\perp}}{(2 \pi)^{3}} \frac{1}{\tilde{q}^{2}} \frac{2 a^{2}}{3}=\frac{C_{R} g_{3 \mathrm{~d}}^{2}}{\tilde{p}_{\perp}^{2}} \frac{C_{A} g_{3 \mathrm{~d}}^{2} a}{4} \frac{8}{3} \frac{\Sigma}{4 \pi} .
$$

\section{$3.4 \quad$ Y-diagrams}

Finally we consider diagrams $E, F, G$. In Coulomb gauge the vertex appearing in $E$ connects three $A_{z}$ propagators. Labeling the lower momentum $p$ and the upper left and right momenta $q$ and $p+q$, we find that $p_{z}=0$ automatically. Applying the vertex Feynman rule (see ref. [29] page 383),

$$
\text { Diagram } E \text { Vertex }=\underline{q}_{z} \tilde{q}_{z}-\widetilde{2 q_{z}}+\tilde{q}_{z} q_{z}=0 .
$$


This is not surprising; for instance, there is certainly no $A_{z}^{3}$ continuum vertex, since $F_{i j}^{2}$ always involves two distinct labels each appearing twice. Diagrams $F$ and $G$ can be computed in a straightforward way using the Feynman rules we have already found for the attachment of lines to the Wilson line, and we find

$$
\begin{aligned}
F & =\frac{C_{R} g_{3 \mathrm{~d}}^{2}}{\tilde{p}_{\perp}^{2}}\left(-2 C_{A} g_{3 \mathrm{~d}}^{2}\right) \int_{-\pi / a}^{\pi / a} \frac{d^{3} q_{\perp}}{(2 \pi)^{3}} \frac{1}{\left(\tilde{q}^{2}\right)^{2}} \tilde{q}_{z} \frac{1}{\tilde{q}_{z}} \\
G & =\frac{C_{R} g_{3 \mathrm{~d}}^{2}}{\tilde{p}_{\perp}^{2}} 2 C_{A} g_{3 \mathrm{~d}}^{2} \int_{-\pi / a}^{\pi / a} \frac{d^{3} q_{\perp}}{(2 \pi)^{3}} \frac{1}{\left(\tilde{q}^{2}\right)^{2}}\left(\tilde{q}_{z} q_{z}\right) \frac{q_{z}}{\tilde{q}_{z}} \\
F+G & =\frac{C_{R} g_{3 \mathrm{~d}}^{2}}{\tilde{p}_{\perp}^{2}} 2 C_{A} g_{3 \mathrm{~d}}^{2} \int_{-\pi / a}^{\pi / a} \frac{d^{3} q_{\perp}}{(2 \pi)^{3}} \frac{1}{\left(\tilde{q}^{2}\right)^{2}}\left(1-\underline{q}_{z}^{2}\right)=\frac{C_{R} g_{3 \mathrm{~d}}^{2}}{\tilde{p}_{\perp}^{2}} \frac{C_{A} g_{3 \mathrm{~d}}^{2} a}{4}\left(-\frac{2}{3} \frac{\Sigma}{4 \pi}\right) .
\end{aligned}
$$

\subsection{Summing it up}

Summing the leading-order and subleading-order contributions of form $1 / p_{\perp}^{2}$, that is, eq. (3.7), eq. (3.8), eq. (3.16), and eq. (3.20), and requiring that the cancellation of $1 / p_{\perp}^{2}$ terms should occur, we find

$$
\begin{aligned}
0 & =\frac{g_{3 \mathrm{~d}}^{2} C_{R}}{p_{\perp}^{2}}\left(1-\frac{Z^{2}}{Z_{\Phi} a}+\frac{g_{3 \mathrm{~d}}^{2} a C_{A}}{4}\left\{-8 \frac{\xi}{4 \pi}-8 \frac{\xi}{4 \pi}-\frac{2}{3} \frac{\Sigma}{4 \pi}+\frac{8}{3} \frac{\Sigma}{4 \pi}-\frac{2}{3} \frac{\Sigma}{4 \pi}\right\}\right) \\
\frac{Z^{2}}{Z_{\Phi} a} & =1+\frac{g_{3 \mathrm{~d}}^{2} a C_{A}}{4}\left(\frac{4}{3} \frac{\Sigma}{4 \pi}-16 \frac{\xi}{4 \pi}\right) .
\end{aligned}
$$

This constitutes our main result.

\section{Discussion}

We have found the 1-loop renormalization factor which should be included in the lattice implementation of the EQCD null Wilson line. Specifically, given the definition of the lattice action found in eq. (2.3) and of the Wilson line operator in eq. (2.5), the ratio of the normalization of the lattice scalar field $\Phi_{\mathrm{L}}$ appearing in the Wilson line to its normalization in the action is given in eq. (3.21), which we repeat for convenience:

$$
\frac{Z^{2}}{Z_{\Phi} a}=1+\frac{g_{3 \mathrm{~d}}^{2} a C_{A}}{4}\left(\frac{4}{3} \frac{\Sigma}{4 \pi}-16 \frac{\xi}{4 \pi}\right)
$$

Using this renormalization in the Wilson line will facilitate faster and more accurate lattice calculations of the infrared contribution to $\hat{q}$ and $C\left(p_{\perp}\right)$. In particular, it eliminates the last source of error (except for $\delta y$, see appendix A) which obstructs a quick and accurate continuum extrapolation in the lattice determination of $C\left(x_{\perp}\right)$.

Structurally the most interesting feature of the calculation is the tendency for diagrams to nearly cancel, when one sums over lines being $A_{z}$ and $\Phi_{\mathrm{L}}=i A_{0}$. This cancellation is broken in the UV because the Wilson line is built out of $\Phi_{\mathrm{L}}$ fields appearing at integer sites and $A_{z}$ fields appearing at half-integer links. Therefore the Wilson line propagator between two like-type fields differs in the UV from that between opposite-type fields. 
There are a few other physically interesting quantities which can be computed with the same methodology as the calculation performed here. It is pointed out in ref. [30] that $\hat{q}$ and its semi-collinear analogue $\hat{q}(\delta E)$ can both be computed as correlation functions of operators separated by adjoint null Wilson lines. The renormalization of the Wilson line found here can be adopted in that problem, though a rather high-loop calculation of UV contributions to the correlator will also be necessary. We leave this to be considered in future work.

\section{Acknowledgments}

We would like to thank Jacopo Ghiglieri, Kari Rummukainen, and Urs Wiedemann for useful discussions. This work was supported in part by the Natural Sciences and Engineering Research Council of Canada. M.D. was supported by the Magnus Ehrnrooth Foundation of Finland.

\section{A Scalar mass and self-coupling renormalization}

Here we write the known 1- and 2-loop renormalizations of the scalar self-couplings and mass in the notation of this paper; and we discuss what would be involved in a full $\mathcal{O}(a)$ (3-loop) determination of $\delta y$, or how it could be avoided by using the lattice to measure the requisite corrections.

The 1-loop renormalization $Z_{g}$ appears already in eq. (3.2). The remaining one-loop renormalizations can be found in ref. [23], and are: ${ }^{9}$

$$
\begin{aligned}
\frac{Z_{4}}{Z_{\Phi}^{2}} & =g_{3 \mathrm{~d}}^{2} a\left(1-g_{3 \mathrm{~d}}^{2} a C_{A}\left[\frac{1}{3} \frac{\Sigma}{4 \pi}+6 \frac{\xi}{4 \pi}\right]\right) \\
\frac{Z_{2}}{Z_{\Phi}} & =g_{3 \mathrm{~d}}^{4} a^{2}\left(1+g_{3 \mathrm{~d}}^{2} a\left[-\frac{C_{A}}{6} \frac{\Sigma}{4 \pi}+\left(-3 C_{A}+\left(N^{2}+1\right) x_{1}+\frac{2 N^{2}-3}{N} x_{2}\right) \frac{\xi}{4 \pi}\right]\right), \\
\delta x_{1} & =g_{3 \mathrm{~d}}^{2} a\left(3+\left(N^{2}+7\right) x_{1}^{2}+2 \frac{2 N^{2}-3}{N} x_{1} x_{2}+\left(3+\frac{9}{N^{2}}\right) x_{2}^{2}\right) \frac{\xi}{4 \pi} \\
\delta x_{2} & =g_{3 \mathrm{~d}}^{2} a\left(N+2 \frac{N^{2}-9}{N} x_{2}^{2}+12 x_{1} x_{2}\right) \frac{\xi}{4 \pi} \\
\delta y_{1 \text { loop }} & =\frac{-1}{g_{3 \mathrm{~d}}^{2} a}\left(2 N+\left(N^{2}+1\right) x_{1}+\frac{2 N^{2}-3}{N} x_{2}\right) \frac{\Sigma}{4 \pi}
\end{aligned}
$$

Here we have departed from our previous pattern of writing everything in terms of Casimirs and dimensions and have specialized to $\mathrm{SU}(N)$ gauge theory, because it is not obvious to us that the form of the quartic interaction we have used can be considered without modification in more general groups.

\footnotetext{
${ }^{9} Z_{2} / g_{3 \mathrm{~d}}^{4} a^{2} Z_{\Phi}$ was called $Z_{m}$ there, and the notation for $x_{1}, x_{2}$, as well as the division between $x$ and $Z_{4}$, was slightly different.
} 
The renormalization of $y$ is known to two loops. Besides the factor $Z_{2}$ included above, one needs

$$
\begin{aligned}
-16 \pi^{2} \delta y_{2} \text { loop }= & \left(\left(N^{2}+1\right) x_{1}+\frac{2 N^{2}-3}{N} x_{2}\right)\left(\frac{N \Sigma^{2}}{2}+N \Sigma \xi-2 N \delta\right) \\
& +N^{2}\left(\frac{7 \Sigma^{2}}{8}-\frac{\Sigma \pi}{6}+\frac{31 \Sigma \xi}{6}+2 \kappa_{1}-\kappa_{4}-4 \rho-4 \delta\right) \\
& +\left(\left(N^{2}+1\right) x_{1}\left(2 N-2 x_{1}\right)+\frac{2 N^{2}-3}{N} x_{2}\left(2 N-4 x_{1}\right)\right. \\
& \left.-\frac{N^{4}-6 N^{2}+18}{N^{2}} x_{2}^{2}\right)\left(\ln \frac{6}{g_{3 \mathrm{~d}}^{2} a}+\zeta-3 \Sigma \xi\right) .
\end{aligned}
$$

Here $\zeta, \delta$, and $4 \rho-2 \kappa_{1}+\kappa_{4}$ are additional constants which are defined in ref. [24]; specifically $\zeta=0.08849, \delta=1.942130$, and $4 \rho-2 \kappa_{1}+\kappa_{4}=-1.968325$. Note that the 1-loop contribution is parametrically $1 / a$ and the two-loop contribution is of order $a^{0}, \ln \left(g_{3 \mathrm{~d}}^{2} a\right)$. Therefore, in a complete $\mathcal{O}(a)$ corrected study, one should also establish the three-loop $\mathcal{O}(a)$ correction to $\delta y$, which will be parametrically of form

$$
\delta y_{3 \text { loop }} \sim g_{3 \mathrm{~d}}^{2} a\left(C_{3} x^{3}+C_{2} x^{2}+C_{1} x+C_{0}\right) .
$$

(Really $C_{3} x^{3}=C_{30} x_{1}^{3} x_{0}^{0}+C_{21} x_{1}^{2} x_{2}+\ldots$ so there are 10 coefficients in all.) The diagrammatic computation of this correction, and particularly of $C_{0}$, appears rather difficult. However, there is an alternative to a diagrammatic computation which could be attempted. The key is that the $\mathrm{SU}(N)$ theory with $N>2$ has a phase transition at some $y_{\text {crit }}\left(x_{1}, x_{2}\right)$ for all values of $x_{1}, x_{2}$. A lattice study, at fixed $x$, can find the critical value of $y$ at a given lattice spacing, $y_{\text {crit }}(a, x)$. One then repeats for several values of $a$, and examines the extrapolation to small $a$. Since all other parameters are known up to $\mathcal{O}\left(a^{2}\right)$ corrections, the only source for $\mathcal{O}(a)$ dependence in $y_{\text {crit }}(a, x)$ is the unknown (x-dependent) $\mathcal{O}(a)$ correction to $\delta y$. If the lattice determination of $y_{\text {crit }}$ is accurate enough to determine the linear in $a$ behavior with precision, this constitutes an evaluation of the terms in eq. (A.7), at a given value of $x$. By repeating for several $x$ values, one can reconstruct all terms. In particular, one can determine $C_{3}$ by studying the theory with the gauge fields switched off, and only scalar fields with quartic interactions. Since much more powerful algorithms exist to study this theory (cluster, worm, multigrid), an accurate determination of $C_{3}$ should be straightforward.

Open Access. This article is distributed under the terms of the Creative Commons Attribution License (CC-BY 4.0), which permits any use, distribution and reproduction in any medium, provided the original author(s) and source are credited.

\section{References}

[1] ALICE collaboration, Elliptic flow of charged particles in Pb-Pb collisions at 2.76 TeV, Phys. Rev. Lett. 105 (2010) 252302 [arXiv:1011.3914] [INSPIRE]. 
[2] ATLAS collaboration, Measurement of the azimuthal anisotropy for charged particle production in $\sqrt{s_{\mathrm{NN}}}=2.76$ TeV lead-lead collisions with the ATLAS detector, Phys. Rev. $\mathbf{C}$ 86 (2012) 014907 [arXiv: 1203.3087] [INSPIRE].

[3] CMS collaboration, Measurement of the elliptic anisotropy of charged particles produced in $\mathrm{PbPb}$ collisions at nucleon-nucleon center-of-mass energy $=2.76 \mathrm{TeV}$, Phys. Rev. $\mathbf{C} \mathbf{8 7}$ (2013) 014902 [arXiv: 1204.1409] [INSPIRE].

[4] M. Luzum and P. Romatschke, Conformal Relativistic Viscous Hydrodynamics: Applications to RHIC results at $\sqrt{s_{\mathrm{NN}}}=200 \mathrm{GeV}$, Phys. Rev. C 78 (2008) 034915 [Erratum ibid. C 79 (2009) 039903] [arXiv:0804.4015] [INSPIRE].

[5] B. Schenke, S. Jeon and C. Gale, Anisotropic flow in $\sqrt{s}=2.76 \mathrm{TeV} P b+P b$ collisions at the LHC, Phys. Lett. B 702 (2011) 59 [arXiv:1102.0575] [INSPIRE].

[6] H. Song, S. Bass and U.W. Heinz, Spectra and elliptic flow for identified hadrons in $2.76 \mathrm{~A}$ $\mathrm{TeV} \mathrm{Pb+Pb}$ collisions, arXiv:1311.0157 [INSPIRE].

[7] CMS collaboration, Studies of jet quenching using isolated-photon+jet correlations in $\mathrm{PbPb}$ and pp collisions at $\sqrt{s_{\mathrm{NN}}}=2.76 \mathrm{TeV}$, Phys. Lett. B 718 (2013) 773 [arXiv:1205.0206] [INSPIRE].

[8] CMS collaboration, Jet momentum dependence of jet quenching in $\mathrm{PbPb}$ collisions at $\sqrt{s_{\mathrm{NN}}}=2.76 \mathrm{TeV}$, Phys. Lett. B 712 (2012) 176 [arXiv:1202.5022] [INSPIRE].

[9] ATLAS collaboration, Measurement of the jet radius and transverse momentum dependence of inclusive jet suppression in lead-lead collisions at $\sqrt{s_{\mathrm{NN}}}=2.76 \mathrm{TeV}$ with the ATLAS detector, Phys. Lett. B 719 (2013) 220 [arXiv:1208.1967] [INSPIRE].

[10] B.G. Zakharov, Fully quantum treatment of the Landau-Pomeranchuk-Migdal effect in QED and QCD, JETP Lett. 63 (1996) 952 [hep-ph/9607440] [INSPIRE].

[11] B.G. Zakharov, Radiative energy loss of high-energy quarks in finite size nuclear matter and quark-gluon plasma, JETP Lett. 65 (1997) 615 [hep-ph/9704255] [INSPIRE].

[12] R. Baier, Y.L. Dokshitzer, S. Peigne and D. Schiff, Induced gluon radiation in a $Q C D$ medium, Phys. Lett. B 345 (1995) 277 [hep-ph/9411409] [INSPIRE].

[13] R. Baier, Y.L. Dokshitzer, A.H. Mueller, S. Peigne and D. Schiff, Radiative energy loss of high-energy quarks and gluons in a finite volume quark-gluon plasma, Nucl. Phys. B 483 (1997) 291 [hep-ph/9607355] [INSPIRE].

[14] P.B. Arnold, Simple Formula for High-Energy Gluon Bremsstrahlung in a Finite, Expanding Medium, Phys. Rev. D 79 (2009) 065025 [arXiv:0808.2767] [INSPIRE].

[15] P.B. Arnold and W. Xiao, High-energy jet quenching in weakly-coupled quark-gluon plasmas, Phys. Rev. D 78 (2008) 125008 [arXiv:0810.1026] [INSPIRE].

[16] S. Caron-Huot, O(g) plasma effects in jet quenching, Phys. Rev. D 79 (2009) 065039 [arXiv:0811.1603] [INSPIRE].

[17] M. Laine and Y. Schröder, Two-loop QCD gauge coupling at high temperatures, JHEP 03 (2005) 067 [hep-ph/0503061] [INSPIRE].

[18] M. Panero, K. Rummukainen and A. Schäfer, A lattice study of the jet quenching parameter, arXiv: 1307.5850 [INSPIRE].

[19] S. Nadkarni, Dimensional Reduction in Finite Temperature Quantum Chromodynamics. 2., Phys. Rev. D 38 (1988) 3287 [INSPIRE]. 
[20] N.P. Landsman, Limitations to Dimensional Reduction at High Temperature, Nucl. Phys. B 322 (1989) 498 [INSPIRE].

[21] E. Braaten and A. Nieto, Free energy of QCD at high temperature, Phys. Rev. D 53 (1996) 3421 [hep-ph/9510408] [INSPIRE].

[22] K. Kajantie, M. Laine, K. Rummukainen and M.E. Shaposhnikov, Generic rules for high temperature dimensional reduction and their application to the standard model, Nucl. Phys. B 458 (1996) 90 [hep-ph/9508379] [INSPIRE].

[23] G.D. Moore, O(a) errors in 3D SU(N) Higgs theories, Nucl. Phys. B 523 (1998) 569 [hep-lat/9709053] [INSPIRE].

[24] M. Laine, Exact relation of lattice and continuum parameters in three-dimensional SU(2) + Higgs theories, Nucl. Phys. B 451 (1995) 484 [hep-lat/9504001] [INSPIRE].

[25] G.D. Moore, Curing O(a) errors in $3 D$ lattice $\mathrm{SU}(2) \times(1)$ Higgs theory, Nucl. Phys. B 493 (1997) 439 [hep-lat/9610013] [INSPIRE].

[26] K. Kajantie, M. Laine, K. Rummukainen and M.E. Shaposhnikov, $3 D \mathrm{SU}(N)+$ adjoint Higgs theory and finite temperature QCD, Nucl. Phys. B 503 (1997) 357 [hep-ph/9704416] [INSPIRE].

[27] K. Kajantie, M. Laine, K. Rummukainen and Y. Schröder, The Pressure of hot QCD up to g6 $\ln (1 / g)$, Phys. Rev. D 67 (2003) 105008 [hep-ph/0211321] [INSPIRE].

[28] M. Laine and Y. Schröder, Quark mass thresholds in QCD thermodynamics, Phys. Rev. D 73 (2006) 085009 [hep-ph/0603048] [INSPIRE].

[29] H.J. Rothe, Lattice gauge theories: An Introduction, World Sci. Lect. Notes Phys. 43 (1992) 1 [World Sci. Lect. Notes Phys. 59 (1997) 1] [World Sci. Lect. Notes Phys. 74 (2005) 1] [World Sci. Lect. Notes Phys. 82 (2012) 1].

[30] J. Ghiglieri, J. Hong, A. Kurkela, E. Lu, G.D. Moore et al., Next-to-leading order thermal photon production in a weakly coupled quark-gluon plasma, JHEP 05 (2013) 010 [arXiv: 1302.5970] [INSPIRE]. 